\title{
On Optimal Regularization Methods for Fractional Differentiation
}

\author{
U. Tautenhahn and R. Gorenflo
}

Dedicated to Prof. L. v. Wolfersdorf on the occasion of his 65 th birthday

\begin{abstract}
In this paper we consider the following fractional differentiation problem: given noisy data $f^{\delta} \in L^{2}(\mathbb{R})$ to $f$, approximate the fractional derivative $u=D_{\beta} f \in L^{2}(\mathbb{R})$ for $\beta>0$, which is the solution of the integral equation of first kind $\left(A_{\beta} u\right)(x)=\frac{1}{\Gamma(\beta)} \int_{-\infty}^{x} \frac{u(t) d t}{(x-t)^{1-\beta}}=f(x)$. Assuming $\left\|f-f^{\delta}\right\|_{L^{2}(\mathbb{R})} \leq \delta$ and $\|u\|_{p} \leq E$ (where $\|\cdot\|_{p}$ denotes the usual Sobolev norm of order $p>0$ ) we answer the question concerning the best possible accuracy for identifying $u$ from the noisy data $f^{\delta}$. Furthermore, we discuss special regularization methods which realize this best possible accuracy.
\end{abstract}

Keywords: Ill-posed problems, fractional differentiation, regularization methods, optimal error bounds

AMS subject classiflcation: Primary $65 \mathrm{~J} 20$, secondary 65 R 30, 65 D 25, 26 A 33

\section{Introduction}

Fractional differentiation problems arise in several contexts and have important applications in science and enginecring (cf., e.g., [6]), and various aspects of it have been treated in the literature of which we cannot give here an exhaustive survey, but let us quote $[3,4,5,8,9,14-16,23]$. In this paper we consider the problem of fractional differentiation in $L^{2}(\mathbb{R})$, i.e., the problem of the numerical computation of the fractional derivative

$$
u(x):=\left(D_{\beta} f\right)(x)=\frac{1}{\Gamma(n+1-\beta)} \frac{d^{n+1}}{d x^{n+1}} \int_{-\infty}^{x} \frac{f(t) d t}{(x-t)^{\beta-n}}
$$

of a function $f$ given in $L^{2}(\mathbb{R})$, where $\beta>0, n$ is a non-negative integer satisfying $n<\beta \leq n+1$ and $\Gamma(\cdot)$ is the Euler gamma function. Our main emphasis is on optimality of error estimates in regularization. It is well known that the problem of fractional differentiation is equivalent to the problem of solving the integral equation of

U. Tautenhahn: Hochschule für Technik, Wirtschaft und Sozialwesen Zittau/Görlitz (FH), FB Mathematik, P.O. Box 261, D-02763 Zittau (u.tautenhahn@htw-zittau.de)

R. Gorenflo: Freie Universität Berlin, Fachbereich Mathematik und Informatik, Arnimallee 2-6, D-14195 Berlin (gorenflo@math.fu-berlin.de) 
the first kind

$$
\left(A_{\beta} u\right)(x):=\frac{1}{\Gamma(\beta)} \int_{-\infty}^{x} \frac{u(t) d t}{(x-t)^{1-\beta}}=f(x), \quad A_{\beta}: D\left(A_{\beta}\right) \subset \dot{L}^{2}(\mathbb{R}) \rightarrow L^{2}(\mathbb{R})
$$

with domain

$$
D\left(A_{\beta}\right)=\left\{\left.u \in L^{2}(\mathbb{R})|| s\right|^{-\beta} U(s) \in L^{2}(\mathbb{R})\right\}
$$

where $U=\mathcal{F} u$ denotes the Fourier transform of $u$ (cf. (1.3)). Equation (1.1) is an Abel integral equation for $0<\beta<1$. Note that for integer $\beta$-values there holds $u(x)=$ $\frac{d^{\beta}}{d x^{\beta}} f(x)$. As a comprehensive reference for fractional differentiation and integration we recommend [16].

We observe that the operator $A_{\beta}: L^{2}(\mathbb{R}) \rightarrow L^{2}(\mathbb{R})$ is densely defined, injective, normal, unbounded, closed and not compact. In addition, the inverse operator $A_{\beta}^{-1}$ is unbounded and the range $R\left(A_{\beta}\right)$ of the operator $A_{\beta}$ is non-closed in $L^{2}(\mathbb{R})$, consequently, problem (1.1) is ill-posed. Problem (1.1) does not have a solution for arbitrary $f \in$ $L^{2}(\mathbb{R})$. Furthermore, the solution $u$ of problem (1.1) (if it exists) does not depend continuously on the data $f$. For general background on ill-posed problems and their regularization we refer to $[1,2,7,10,12,22,25]$.

Let us illustrate the properties that both $A_{\beta}$ and $A_{\beta}^{-1}$ are unbounded, by the following proposition.

Proposition 1.1. For every pair $(u, f)$ for which $A_{\beta} u=f$ holds there exist sequences $\left(u_{n}\right)$ and $\left(f_{n}\right)$ such that $(\mathrm{a})$ and $(\mathrm{b})$ hold:

(a) $A_{\beta} u_{n}=f_{n}, u_{n} \rightarrow u$ and $f_{n}+f=A_{\beta} u$.

(b) $A_{\beta} u_{n}=f_{n}, f_{n} \rightarrow f=A_{\beta} u$ and $u_{n} \not u$.

Proof. Since $A_{\beta}$ is linear it is sufficient to verify the statements (a) and (b) for $f=0, u=0$. We sketch the proof for the special case $\beta=1$. In order to verify (a) we choose:

$$
f_{n}=\frac{x}{n^{1 / 4}\left(1+|x|^{(3 n+1) /(2 n)}\right)} .
$$

Then the equation $A_{\beta} u_{n}=f_{n}$ with $\beta=1$ has the (unique) solution

$$
u_{n}(x)=f_{n}^{\prime}(x)=\frac{1-\frac{n+1}{2 n}|x|^{(3 n+1) /(2 n)}}{n^{1 / 4}\left(1+|x|^{(3 n+1) /(2 n)}\right)^{2}}
$$

and both $f_{n}$ and $u_{n}$ are in $L^{2}(\mathbb{R})$. Now it can be shown that

$$
\left\|f_{n}\right\|_{L^{2}(\mathbb{R})} \sim n^{1 / 4} \rightarrow \infty \text { and }\left\|u_{n}\right\|_{L^{2}(\mathbb{R})} \sim n^{-1 / 4} \rightarrow 0
$$

for $n \rightarrow \infty$, which verifies (a). In order to prove (b) we choose $f_{n}=e^{-n x^{2}}$. Then the equation $A_{\beta} u_{n}=f_{n}$ with $\beta=1$ has the (unique) solution $u_{n}=f_{n}^{\prime}=-2 n x e^{-n x^{2}}$ and both $f_{n}$ and $u_{n}$ are in $L^{2}(\mathbb{R})$. Since

$$
\left\|f_{n}\right\|_{L^{2}(\mathbb{R})}=\left(\frac{\pi}{2 n}\right)^{1 / 4} \rightarrow 0 \quad \text { and } \quad\left\|u_{n}\right\|_{L^{2}(\mathbb{R})}=\left(\frac{\pi n}{2}\right)^{1 / 4} \rightarrow \infty
$$

for $n \rightarrow \infty$ we obtain (b) 
Since the data $f$ in problem (1.1) are generally based on (physical) observations and not known with complete accuracy (the available noisy data are $f^{\delta} \in L^{2}(\mathbb{R})$ with $\left.\left\|f-f^{\delta}\right\|_{L^{2}(\mathbb{R})} \leq \delta\right)$, for a stable numerical approximation of the solution $u$ of problem (1.1) some regularization technique has to be applied, which provides a sequence of approximations $u_{\alpha}^{\delta}=R_{\alpha} f^{\delta}$ with $u_{\alpha}^{\delta} \rightarrow u$ as $\delta \rightarrow 0$ under proper choice of the regularization parameter $\alpha$. Hence, regularized solutions $u_{\alpha}^{\delta}$ depend continuously on the data. However, as is typical for ill-posed problems, the convergence of $u_{\alpha}^{\delta}$ to $u$ can be arbitrarily slow if we do not impose additional a priori restrictions on the unknown solution $u$ (or on the 'exact' data $f$, respectively). Quantitative a priori restrictions that will work in different ill-posed problems (and enable us to overcome the ill-posedness) consist in imposing a bound $E$ on the (unknown) solution and a finite number of its derivatives.

Let us describe our quantitative a priori information concerning $u$ in more detail. We introduce the Sobolev scale $\left(H^{r}\right)_{r \in \mathbb{R}^{+}}$of positive real order $r$ (cf. [11]) according to

$$
H^{0}=H=L^{2}(\mathbb{R}) \quad \text { and } \quad H^{r}=\left\{v \in H\|\| v \|_{r}<\infty\right\}
$$

where

$$
\|v\|_{r}=\left\|\left(1+s^{2}\right)^{\frac{r}{2}} V(s)\right\|_{L^{2}(\mathbb{R})}=\left(\int_{-\infty}^{+\infty}\left(1+s^{2}\right)^{r}|V(s)|^{2} d s\right)^{\frac{1}{2}} \quad\left(r \in \mathbb{R}^{+}\right)
$$

is the norm in $H^{r}$ and $V=\mathcal{F} v$ denotes the Fourier transform of $v$ given by

$$
V(s)=(\mathcal{F} v)(s)=\frac{1}{\sqrt{2 \pi}} \int_{-\infty}^{+\infty} v(x) e^{-i s x} d x \quad(s \in \mathbb{R}) .
$$

In working with the Fourier transform we will consistently use $s$ for the transform variable, and for transforms of functions we will use the corresponding capital letters, e.g., $F=\mathcal{F} f$. By $\|\cdot\|$ and $(\cdot, \cdot)$ we denote the norm and the inner product in $H^{0}=$ $H=L^{2}(\mathbb{R})$, respectively. Let us mention here one further important convention for this paper: If we work with certain densely defined operators $A$ and $A^{*} A$, respectively, and if we write down elements $A u$ and $\psi\left(A^{*} A\right) v$ with certain operator functions $\psi(\cdot)$, then consistently we will assume in these cases that $u \in D(A)$ and $v \in D\left(\psi\left(A^{*} A\right)\right)$, respectively, without mention this restriction explicitly.

Now we require for problem (1.1) the a priori smoothness condition concerning the unknown solution $u$ according to

$$
u \in M_{p, E}=\left\{u \in H \mid\|u\|_{p} \leq E\right\}
$$

where $p>0$. In the case $p=1$ condition (1.4) means that $\|u\|_{1}=\left\{\|u\|^{2}+\left\|u_{x}\right\|^{2}\right\}^{1 / 2} \leq$ $E$, which follows from Parseval's relation $\|v\|=\|V\|$ and the differentiation theorem for the Fourier transform. Likewise the set $M_{p, E}$ for $p>0$ an integer consists of those functions for which the derivatives of order less or equal $p$ are bounded in the $L^{2}(\mathbb{R})$ norm. The larger $p$, the more restrictive is assumption (1.4). 
Any operator $R: H \rightarrow H$ can be considered as a special method for identifying the solution $u$ of problem (1.1) from noisy data $f^{\delta} \in H$; the approximate solution is then given by $R f^{\delta}$. We introduce the worst case error $\Delta(\delta, R)$ for identifying $u$ from $f^{\delta} \in H$ under the conditions $\left\|f-f^{\delta}\right\| \leq \delta$ and $u \in M_{p, E}$ by

$$
\Delta(\delta, R)=\sup \left\{\left\|R f^{\delta}-u\right\| \mid u \in M_{p, E}, f^{\delta} \in H,\left\|A_{\beta} u-f^{\delta}\right\| \leq \delta\right\} .
$$

This worst case error characterizes the maximal error of an arbitrary method $R$ if the solution $u$ varies in the set $M_{p, E}$ given in (1.4). Now we ask the question concerning the magnitude of the worst case error $\Delta(\delta, R)$ for 'optimal' methods $R: H \rightarrow H$ that minimize the worst case error (1.5) over all methods $R: H \rightarrow H$. In Section 2 we discuss a general formula for the best possible worst case error

$$
\omega(\delta):=\inf _{R} \Delta(\delta, R)
$$

which shows us in which kind this best possible worst case error depends on the noise level $\delta$. In Section 3 we apply this general optimality result to the problem of fractional differentiation and prove that for $\delta \rightarrow 0$

$$
\omega(\delta)=E^{\frac{\rho}{p+\beta}} \delta^{\frac{p}{p+\beta}}(1+o(1)) .
$$

In Section 4 we discuss special regularization methods for the problem of fractional differentiation. We construct regularized approximations $u_{\alpha}^{\delta}=R_{\alpha} f^{\delta}$ to the unknown $u$ that guarantee 'optimal' error bounds $\left\|u_{\alpha}^{\delta}-u\right\| \leq \omega(\delta)$. Hence, the constructed approximations $u_{\alpha}^{\delta}$ are as accurate as possible in terms of the given information $\| f-$ $f^{\delta} \| \leq \delta$ and (1.4). These constructed approximations require the knowledge of the smoothness parameter $p$, the a priori bound $E$ and the noise level $\delta$. In Section 5 we generalize the results of Sections 3 and 4 . We prove that

$$
\omega_{q}(\delta)=E^{\frac{q+\beta}{p+\beta}} \delta^{\frac{p-q}{p+\beta}}(1+o(1))
$$

is the best possible worst case error with respect to the $H^{q}$-norm, and we construct regularization methods that guarantee the optimal error bound $\left\|u_{\alpha}^{\delta}-u\right\|_{q} \leq \omega_{q}(\delta)$.

\section{Optimal error bounds and regularization methods}

In this section we consider an arbitrary ill-posed inverse problem

$$
A u=f
$$

where $A: H_{1} \rightarrow H_{2}$ is a linear injective, not necessarily bounded, but densely defined and closed operator between infinite dimensional Hilbert spaces $H_{1}$ and $H_{2}$. We assume throughout this section that the inverse operator $A^{-1}$ is unbounded, hence, the range $R(A)$ of $A$ is non-closed. Furthermore, we assume that $f^{\delta} \in H_{2}$ are the available noisy data with $\left\|f-f^{\delta}\right\| \leq \delta$. Any operator $R: H_{2} \rightarrow H_{1}$ can be considered as a special 
method for 'approximately' solving (2.1). The approximate solution to (2.1) is then given by $R f^{\delta}$.

Let $M \subset H_{1}$ be an arbitrary set. We introduce the worst case error $\Delta(\delta, R)$ for identifying $u$ from $f^{\delta} \in H_{2}$ under the assumptions $\left\|f-f^{\delta}\right\| \leq \delta$ and $u \in M$ according to

$$
\Delta(\delta, R)=\sup \left\{\left\|R f^{\delta}-u\right\| \mid u \in M, f^{\delta} \in H_{2},\left\|A u-f^{\delta}\right\| \leq \delta\right\} .
$$

This worst case error characterizes the maximal error of the method $R$ if the solution $u$ of problem (2.1) varies in the set $M$. Parameter dependent methods $R=R_{\delta}$ are called

(i) optimal on the set $M$ if $\Delta\left(\delta, R_{\delta}\right)=\inf _{R: H_{2} \rightarrow H_{1}} \Delta(\delta, R)$

(ii) order optimal on the set $M$ if $\Delta\left(\delta, R_{\delta}\right) \leq c \inf _{R: H_{2} \rightarrow H_{1}} \Delta(\delta, R)$ with $c \geq 1$.

For a general discussion of optimality of parameter dependent regularization methods $R_{\delta}$ in the special case that the operator $A$ is bounded and that the set $M$ is given by

$$
M=\left\{u \in H_{1} \mid u=\left(A^{*} A\right)^{\frac{p}{2}} v,\|v\| \leq E, p>0\right\}
$$

we refer to $[12,17,18,24,25]$; concerning order optimality we refer to $[1,2,12,25]$.

In this section we discuss some optimality results if the set $M$ is given by

$$
M_{\varphi, E}=\left\{u \in H_{1} \mid u=\left[\varphi\left(A^{*} A\right)\right]^{\frac{1}{2}} v,\|v\| \leq E\right\}
$$

where the operator function $\varphi\left(A^{*} A\right)$ is well defined via the spectral representation

$$
\varphi\left(A^{*} A\right)=\int_{0}^{a} \varphi(\lambda) d E_{\lambda} .
$$

Here $A^{*} A=\int_{0}^{a} \lambda d E_{\lambda}$ is the spectral decomposition of $A^{*} A,\left\{E_{\lambda}\right\}$ denotes the spectral family of the operator $A^{*} A$ and $a$ is a constant satisfying $\left\|A^{*} A\right\| \leq a$, with $a=\infty$ if $A^{*} A$ is unbounded. In the case that $A: L^{2}(\mathbb{R}) \rightarrow L^{2}(\mathbb{R})$ is a multiplication operator, $A u(s)=a(s) u(s)$, the operator function $\varphi\left(A^{*} A\right)$ attains the form

$$
\varphi\left(A^{*} A\right) u(s)=\varphi\left(|a(s)|^{2}\right) u(s)
$$

The optimality results which will be discussed in the sequel of this chapter have been obtained in the case of bounded operators $A$ in [19]. If $A$ is densely defined and closed, the same properties are valid for the adjoint $A^{*}$, and for the operator $A^{*} A$ there exists a unique spectral decomposition. Since the proofs for the optimality results in [19] are based on spectral theory, they can be extended with small modifications to the case of operator equations (2.1) with (unbounded) densely defined and closed operators $A$ and will be omitted here.

Let us motivate the practical relevance of restricting attention to the general source set (2.3). In various ill-posed problems (2.1) additional quantitative a priori restriction 
is given by a certain smoothness of the unknown solution $u \in H_{1}$. Such smoothness conditions can be reformulated into equivalent conditions $u \in M_{\varphi, E}$ with certain functions $\varphi$ which generally are not of the form $\varphi(\lambda)=\lambda^{p}$. Hence, the set $M_{\varphi, E}$ does not coincide with the above set $M$ in general, and the general optimality results known for the above set $M$ cannot be applied. Examples where $\varphi$ differs from the standard form $\varphi(\lambda)=\lambda^{p}$ arise, e.g., in heat equation problems backward in time (cf. [19]), in non-characteristic Cauchy problems for elliptic partial differential equations (cf. [20]) or in sideways heat equation problems (cf. [21]). For our fractional differentiation problem of Section 1 we will see in Section 3 that the specific a priori restriction (1.4) concerning the unknown solution can be reformulated into an equivalent condition (2.3) with a special function $\varphi$. This reformulation enables us to apply optimality results which we discuss in the sequel of this section.

In order to derive explicit (best possible) error bounds for the worst case error $\Delta(\delta, R)$ defined in (2.2) and in order to obtain optimality results for special regularization methods we assume that the function $\varphi$ in (2.3) satisfies the following assumption.

Assumption 2.1. The function $\varphi:(0, a] \rightarrow(0, \infty)$ in $(2.3)$ (where $a$ is a constant with $\left.\left\|A^{*} A\right\| \leq a\right)$ is continuous and satisfies following properties:

(i) $\lim _{\lambda \rightarrow 0} \varphi(\lambda)=0$.

(ii) $\varphi(\lambda)$ is strictly monotonically increasing on $(0, a]$.

(iii) $\rho(\lambda)=\lambda \varphi^{-1}(\lambda):(0, \varphi(a)] \rightarrow(0, a \varphi(a)]$ is convex.

The following theorem gives us a general formula for the best possible worst case error inf $R \Delta(\delta, R)$. The proof of this formula can be found in [19] for the special case of bounded operators $A$ and follows some ideas given in [13: Theorem 2.10] and [24], where the case $\varphi(\lambda)=\lambda^{p}(p>0)$ is treated.

Theorem 2.2. Let $M_{\varphi, E}$ be given by (2.3), let Assumption 2.1 be satisfied and let $\delta^{2} / E^{2} \in \sigma\left(A^{*} A \varphi\left(A^{*} A\right)\right)$ where $\sigma\left(A^{*} A\right)$ denotes the spectrum of the operator $A^{*} A$. Then

$$
\inf _{R} \Delta(\delta, R)=E \sqrt{\rho^{-1}\left(\delta^{2} / E^{2}\right)}
$$

Note that the condition $\delta^{2} / E^{2} \in \sigma\left(A^{*} A \varphi\left(A^{*} A\right)\right)$ can only hold (for sufficiently small $\delta)$ if the problem (2.1) is ill-posed. For well-posed problems (2.1) condition $\delta^{2} / E^{2} \in$ $\sigma\left(A^{*} A \varphi\left(A^{*} A\right)\right)$ can never hold for sufficiently small $\delta$, hence this condition excludes the class of well-posed problems.

Furthermore, in [19] special regularization methods have been constructed which realize the error bound given on the right-hand side of (2.4) in the case of bounded operators $A$. These results can also be extended to the case of (unbounded) densely defined and closed operators. In the following considerations we discuss three special methods, a special variant of the method of generalized Tikhonov regularization and two variants of the method of generalized spectral decomposition.

In our method of generalized Tikhonov regularization a regularized approximation $u_{\alpha}^{\delta}$ is determined by solving the minimization problem

$$
\min _{u \in H_{1}} J_{\alpha}(u), \quad J_{\alpha}(u)=\left\|A u-f^{\delta}\right\|^{2}+\alpha\left\|\left[\varphi\left(A^{*} A\right)\right]^{-\frac{1}{2}} u\right\|^{2}
$$


Due to Assumption 2.1, Morozov's complementation condition (cf., e.g. [2])

$$
\|A u\|^{2}+\left\|\left[\varphi\left(A^{*} A\right)\right]^{-\frac{1}{2}} u\right\|^{2} \geq \gamma\|u\|^{2}
$$

is satisfied with $\gamma>0$. Consequently, problem (2.5) has a unique solution which is given by

$$
u_{\alpha}^{\delta}=A^{*}\left(A A^{*}+\alpha\left[\varphi\left(A A^{*}\right)\right]^{-1}\right)^{-1} f^{\delta} \text {. }
$$

This method appears to be optimal on the set $M_{\varphi, E}$ given by (2.3) provided the regularization parameter $\alpha$ is chosen properly.

Theorem 2.3. Let $M_{\varphi, E}$ be given by (2.3), let Assumption 2.1 be satisfied, $\varphi=$ $\varphi(\lambda)$ be twice differentiable, $\rho=\rho(\lambda)$ be strictly convex and $\delta^{2} / E^{2} \leq a \varphi(a)$. If the regularization parameter $\alpha$ is chosen optimally as

$$
\alpha=\frac{\lambda_{0}}{\varphi^{-1}\left(\lambda_{0}\right) \varphi^{\prime}\left(\varphi^{-1}\left(\lambda_{0}\right)\right)}\left(\frac{\delta}{E}\right)^{2} \quad \text { with } \quad \lambda_{0}=\rho^{-1}\left(\frac{\delta^{2}}{E^{2}}\right)
$$

then for the Tikhonov regularized solution $u_{\alpha}^{\delta}=R_{\alpha} f^{\delta}$ defined by (2.5) the error estimate

$$
\Delta\left(\delta, R_{\alpha}\right) \leq E \sqrt{\rho^{-1}\left(\delta^{2} / E^{2}\right)}
$$

holds, which is optimal in case $\delta^{2} / E^{2} \in \sigma\left(A^{*} A \varphi\left(A^{*} A\right)\right)$.

Proof. Let us sketch the ideas of proof. From [24: Lemma 2.2] we have

$$
\begin{aligned}
\Delta\left(\delta, R_{\alpha}\right) & =\inf _{0<\xi<1}\left\|\frac{E^{2}}{\xi}\left(I-R_{\alpha} A\right) \varphi\left(A^{*} A\right)\left(I-R_{\alpha} A\right)^{*}+\frac{\delta^{2}}{1-\xi} R_{\alpha} R_{\alpha}^{*}\right\|^{\frac{1}{2}} \\
& \leq \inf _{0<\xi<1} \sup _{\lambda>0}\left\{\frac{E^{2} \alpha^{2} \varphi(\lambda)}{\xi[\lambda \varphi(\lambda)+\alpha]^{2}}+\frac{\delta^{2} \lambda \varphi^{2}(\lambda)}{(1-\xi)[\lambda \varphi(\lambda)+\alpha]^{2}}\right\}^{\frac{1}{2}} .
\end{aligned}
$$

Now we search for a stationary point $\left(\alpha_{s}, \xi_{s}, \lambda_{s}\right)$ of the expression $\{\ldots\}$ as a function of $\alpha, \xi$ and $\lambda$ and find $\alpha_{s}$ as given in (2.7) and $\xi_{s}=E^{2} \alpha_{s} /\left(\delta^{2}+E^{2} \alpha_{s}\right)$. We substitute $\alpha_{s}$ and $\xi_{s}$ into the expression $\{\ldots\}$ and prove that the remaining expression as a function of $\lambda>0$ is bounded by $E \sqrt{\rho^{-1}\left(\delta^{2} / E^{2}\right)}$

Now we discuss two different spectral methods which are both optimal on the set $M_{\varphi, E}$ given by (2.3) provided the regularization parameter $\alpha$ is chosen properly.

The first spectral method is characterized by

$$
u_{\alpha}^{\delta}=A^{*} g_{\alpha}\left(A A^{*}\right) f^{\delta} \quad \text { with } g_{\alpha}(\lambda)= \begin{cases}\frac{1}{\lambda} & \text { for } \lambda \geq \alpha \\ \frac{1}{\alpha} & \text { for } \lambda<\alpha .\end{cases}
$$

For-this method the following optimality result can be established (see [19] for the special case of bounded operators $A$ ), where the ideas of proof are similar to those for proving Theorem 2.3. 
Theorem 2.4. Let $M_{\varphi, E}$ be given by (2.3), let Assumption 2.1 be satisfied, $\varphi=$ $\varphi(\lambda)$ be twice differentiable, $\rho=\rho(\lambda)$ be strictly convex and $\delta^{2} / E^{2} \leq a \varphi(a)$. If the regularization parameter $\alpha$ is chosen optimally as

$$
\alpha=\frac{\varphi\left(\lambda_{0}\right)+\lambda_{0} \varphi^{\prime}\left(\lambda_{0}\right)}{\varphi^{\prime}\left(\lambda_{0}\right)} \quad \text { with } \lambda_{0} \varphi\left(\lambda_{0}\right)=\left(\frac{\delta}{E}\right)^{2}
$$

then for the regularized solution $u_{\alpha}^{\delta}=R_{\alpha} f^{\delta}$ defined by (2.9) the error estimate (2.8) holds. This error estimate is optimal in the case $\delta^{2} / E^{2} \in \sigma\left(A^{*} A \varphi\left(A^{*} A\right)\right)$.

In the second spectral method a regularized approximation $x_{\alpha}^{\delta}$ is determined by

$$
u_{\alpha}^{\delta}=A^{*} g_{\alpha}\left(A A^{*}\right) f^{\delta} \quad \text { with } g_{\alpha}(\lambda)= \begin{cases}\frac{1}{\lambda} & \text { for } \lambda \geq \alpha \\ \frac{1}{\sqrt{\alpha \lambda}} & \text { for } \lambda<\alpha .\end{cases}
$$

For this method the following optimality result holds (see [19] for the special case of bounded operators $A$ ). The ideas of proof are similar to those used in the proof of Theorem 2.3.

Theorem 2.5. Let $M_{\varphi, E}$ be given by (2.3), let Assumption 2.1 be satisfied, $\varphi=$ $\varphi(\lambda)$ be twice differentiable, $\delta^{2} / E^{2} \leq a \varphi(a)$ and let

$$
\varphi(\lambda) \varphi^{\prime}(\lambda)+2 \lambda \varphi(\lambda) \varphi^{\prime \prime}(\lambda)-3 \lambda\left[\varphi^{\prime}(\lambda)\right]^{2}<0
$$

hold. If the regularization parameter $\alpha$ is chosen optimally as

$$
\alpha=\frac{1}{\lambda_{0}}\left(\frac{\varphi\left(\lambda_{0}\right)+\lambda_{0} \varphi^{\prime}\left(\lambda_{0}\right)}{\varphi^{\prime}\left(\lambda_{0}\right)}\right)^{2} \quad \text { with } \lambda_{0} \varphi\left(\lambda_{0}\right)=\left(\frac{\delta}{E}\right)^{2}
$$

then for the regularized solution $u_{\alpha}^{\delta}=R_{\alpha} f^{\delta}$ defined by (2.11) the error estimate (2.8) holds. This error estimate is optimal in the case $\delta^{2} / E^{2} \in \sigma\left(A^{*} A \varphi\left(A^{*} A\right)\right)$.

\section{Optimal error bounds for fractional differentiation}

In this section we consider the fractional differentiation problem (1.1) and treat the question concerning the best possibe worst case error (1.6) for identifying $u$ from noisy data $f^{\delta} \in H=L^{2}(\mathbb{R})$ provided $\left\|f-f^{\delta}\right\| \leq \delta$ and $u \in M_{p, E}$ hold where $M_{p, E}$ is given by (1.4). Let us consider the operator equation (1.1) with the linear operator $A_{\beta}: H \rightarrow H$. Obviously, this equation is equivalent to the operator equation

$$
\widehat{A}_{\beta} U=F \quad \text { with } \widehat{A}_{\beta}=\mathcal{F} A_{\beta} \mathcal{F}^{-1}
$$

where $\mathcal{F}: L^{2}(\mathbb{R}) \rightarrow L^{2}(\mathbb{R})$ is the (unitary) Fourier operator (cf. (1.3)) that maps any $L^{2}(\mathbb{R})$-function $v=v(x)$ into it's Fourier transform $V=V(s)$. Transforming problem (1.1) we obtain

$$
(i s)^{-\beta} U(s)=F(s)
$$


(cf., e.g., [6]), consequently,

$$
\widehat{A}_{\beta}=(i s)^{-\beta}=|s|^{-\beta} e^{-i \beta \operatorname{sgn}(s) \frac{\pi}{2}}
$$

which shows us that $\hat{A}_{\beta}: H \rightarrow H$ in problem (3.1) is a linear, injective, normal and unbounded operator (multiplication operator), where also the inverse $\widehat{A}_{\beta}^{-1}$ is unbounded. Since $\widehat{A}_{\beta}^{*}=|s|^{-\beta} e^{i \beta \operatorname{sgn}(s) \frac{\pi}{2}}$ we have

$$
\widehat{A}_{\beta}^{*} \widehat{A}_{\beta}=\widehat{A}_{\beta} \hat{A}_{\beta}^{*}=|s|^{-2 \beta},
$$

hence the spectrum of this operator is given by $\sigma\left(\widehat{A}_{\beta}^{*} \widehat{A}_{\beta}\right)=[0, \infty)$. Since $\widehat{A}_{\beta}^{*} \hat{A}_{\beta}$ tends to zero as $|s|$ tends to infinity we realize that problem (3.1) is ill-posed. We call problem (3.1)

mildly ill-posed for $0<\beta \leq 1$ and

moderately ill-posed for $1<\beta<\infty$.

Obviously, the ill-posedness becomes worse as $\beta$ increases. High frequency perturbations of the data will be blown up more and more for increasing $\beta$ and the corresponding solutions $\mathcal{F}^{-1} \widehat{A}_{\beta}^{-1} F^{\delta}$ (if they exist) will indicate strong and undesired oscillations.

The smoothness condition (1.4) can also be transformed into an equivalent condition in the frequency domain. From (1.2) we have that condition (1.4) is equivalent to the condition

$$
U \in \widehat{M}_{p, E}=\left\{U \in H \mid\left\|\left(1+\cdot^{2}\right)^{\frac{2}{2}} U\right\| \leq E\right\}
$$

where dot replaces the variable $s$. This condition can be reformulated into an equivalent condition with a set of the structure (2.3).

Proposition 3.1. Consider the operator equation (3.1). Then the set $\widehat{M}_{p, E}$ given in (3.5) is equivalent to the general source set

$$
\widehat{M}_{\varphi, E}=\left\{U \in H \mid\left\|\left[\varphi\left(\widehat{A}_{\beta}^{*} \widehat{A}_{\beta}\right)\right]^{-\frac{1}{2}} U\right\| \leq E\right\}
$$

where $\varphi=\varphi$ is given (in parameter representation) by

$$
\left.\begin{array}{l}
\lambda=r^{-\beta} \\
\varphi=(1+r)^{-p}
\end{array}\right\} \quad(0 \leq r<\infty) .
$$

Proof. We compare (3.5) and (3.6) and obtain

$$
\varphi\left(\widehat{A}_{\beta}^{*} \hat{A}_{\beta}\right)=\left(1+s^{2}\right)^{-p} .
$$

From this representation and (3.4) we obtain that $\varphi$ is given (in parameter representation) by $\lambda=|s|^{-2 \beta}, \varphi=\left(1+s^{2}\right)^{-p} \quad(s \in \mathbb{R})$. We substitute $s^{2}=r$ and obtain (3.7)

In our following considerations we discuss properties of the function $\varphi=\varphi(\lambda)(\lambda \in$ $(0, \infty)$ ) which is given (in parameter representation) by (3.7). 
Proposition 3.2. The function $\varphi$ defined by (3.7) is continuous and satisfies the following properties:

(i) $\lim _{\lambda \rightarrow 0} \varphi(\lambda)=0$.

(ii) $\varphi$ is strictly monotonically increasing.

(iii) $\rho(\lambda)=\lambda \varphi^{-1}(\lambda)$ is strictly monotonically increasing as well as strictly convex and possesses the parameter representation

$$
\left.\begin{array}{l}
\lambda=(1+r)^{-p} \\
\rho=r^{-\beta}(1+r)^{-p}
\end{array}\right\} \quad(0 \leq r<\infty) .
$$
tation

(iv) $\rho^{-1}$ is strictly monotonically increasing and possesses the parameter represen-

$$
\left.\begin{array}{rl}
\lambda & =r^{-\beta}(1+r)^{-p} \\
\rho^{-1} & =(1+r)^{-p}
\end{array}\right\} \quad(0 \leq r<\infty) .
$$

(v) For the inverse function $\rho^{-1}$ of $\rho$,

$$
\rho^{-1}(\lambda)=\lambda^{\frac{p}{p+\beta}}(1+o(1)) \quad \text { for } \lambda \rightarrow 0
$$

holds.

Proof. Since the function $\lambda(r)$ from (3.7) is strictly monotonically decreasing with $\lim _{r \rightarrow \infty} \lambda(r)=0$ we have $\lim _{\lambda \rightarrow 0} \varphi(\lambda)=\lim _{r \rightarrow \infty} \varphi(r)=0$, hence (i) is proved. Since $\dot{\lambda}(r)<0$ and $\dot{\varphi}(r)<0$ we have $\varphi^{\prime}(\lambda)=\frac{\dot{\varphi}(r)}{\lambda(r)}>0$ which gives (ii). From (i) and (ii) it follows that $\varphi^{-1}(\lambda)$ is strictly monotonically increasing, consequently, $\rho(\lambda)$ is strictly monotonically increasing, where (3.9) follows from the parameter representation of $\varphi^{-1}$ which is given by

$$
\left.\begin{array}{rl}
\lambda & =(1+r)^{-p} \\
\varphi^{-1} & =r^{-\beta}
\end{array}\right\} \quad(0 \leq r<\infty) .
$$

Now let us discuss the convexity of $\rho$. From $\rho^{\prime \prime}=[\ddot{\rho} \dot{\lambda}-\dot{\rho} \ddot{\lambda}] / \dot{\lambda}^{3}$ and $\dot{\lambda}<0$ we obtain that $\rho^{\prime \prime}>0$ is equivalent to $\ddot{\rho} \dot{\lambda}<\dot{\rho} \ddot{\lambda}$. By elementary calculations it can be shown that this inequality is satisfied provided $p>0$ and $\beta>0$ hold, hence, $\rho$ is strictly convex. Now (iv) is a direct consequence of (iii), and (v) follows from (iv)

Now we are in a position to formulate our main result of this section concerning the best possible worst case error $\omega(\delta)$ defined in (1.6) for identifying the solution $u$ of problem (1.1) from noisy data $f^{\delta} \in L^{2}(\mathbb{R})$ under the conditions $\left\|f-f^{\delta}\right\| \leq \delta$ and $u \in M_{p, E}$ where the set $M_{p, E}$ is given by (1.4).

Theorem 3.3. Let $\delta^{2} / E^{2}<\infty, p>0$ and $\beta>0$. Then the stability result

$$
\begin{aligned}
\omega(\delta) & =E\left(1+r_{0}\right)^{-\frac{2}{2}} \\
& =E^{\frac{\rho}{p+\sigma}} \delta^{\frac{p}{p+o}}(1+o(1)) \quad \text { for } \delta \rightarrow 0
\end{aligned}
$$


holds (Hölder stability) where $r_{0}$ is the (unique) solution of the equation

$$
r^{-\beta}(1+r)^{-p}=\left(\frac{\delta}{E}\right)^{2}
$$

Proof. Since the Fourier operator $\mathcal{F}$ is unitary (i.e. $\mathcal{F}^{-1}=\mathcal{F}^{*}$ ) we have for the worst case error (1.6)

$$
\omega(\delta)=\widehat{\omega}(\delta):=\inf _{\widehat{R}} \sup \left\{\left\|\widehat{R} F^{\delta}-U\right\| \mid F^{\delta} \in H,\left\|\dot{F}-F^{\delta}\right\| \leq \delta, U \in \widehat{M}_{p, E}\right\} .
$$

Now we apply Theorem 2.2 to our problem (3.1). Due to (3.14), formulae (3.12) and (3.13) follow from (3.10) and (3.11) provided Assumption 2.1 with $A=\widehat{A}_{\beta}$ and $\varphi$ given by (3.7) as well as

$$
\left(\frac{\delta}{E}\right)^{2} \in \sigma\left(\widehat{A}_{\beta}^{*} \widehat{A}_{\beta} \varphi\left(\hat{A}_{\beta}^{*} \hat{A}_{\beta}\right)\right)
$$

hold. Assumption 2.1 is verified by Proposition 3.2. In order to verify (3.15) we use (3.4) and (3.8) and obtain that

$$
\sigma\left(\widehat{A}_{\beta}^{*} \widehat{A}_{\beta} \varphi\left(\widehat{A}_{\beta}^{*} \widehat{A}_{\beta}\right)\right)=\sigma\left(|s|^{-2 \beta}\left(1+s^{2}\right)^{-p}\right)=[0, \infty)
$$

holds. Consequently, (3.15) reduces to $\delta^{2} / E^{2}<\infty$, and the proof is complete

\section{Optimal regularization methods}

In Section 3 we have proved that the best possible worst case error for identifying $u$ in equation (1.1) from noisy data $f^{\delta} \in L^{2}(\mathbb{R})$ under the conditions $\left\|f-f^{\delta}\right\| \leq \delta$ and $u \in M_{p, E}$ (with $M_{p, E}$ given by (1.4)) is given by (3.12). From this result we conclude that under the above conditions there do not exist any methods $R: H \rightarrow H$ which guarantee an error bound for $\left\|R f^{\delta}-u\right\|$ which is smaller than that given on the righthand side of (3.12).

In this section we consider the method of generalized Tikhonov regularization and two variants of the method of generalized spectral decomposition, apply these methods to the problem of fractional differentiation and show how to choose the regularization parameter such that all three methods guarantee the optimal error bound given by (3.12). These optimality results will be obtained by applying Theorems $2.3-2.5$ to our transformed problem (3.1). All three theorems yield 'optimal' regularized approximations $U_{\alpha}^{\delta}$ in the frequency domain, and due to the Parseval relation $\left\|U_{\alpha}^{\delta}-U\right\|=\left\|u_{\alpha}^{\delta}-u\right\|$ it follows that the elements $u_{\alpha}^{\delta}=\mathcal{F}^{-1} U_{\alpha}^{\delta}$ are then 'optimal' regularized approximations in the original domain.

For the problem of fractional differentiation we obtain together with (3.3) and (3.8) that the method of generalized Tikhonov regularization (2.5) applied to our problem (3.1) in the frequency domain consists in the determination of a regularized approximation $U_{\alpha}^{\delta}=U_{\alpha}^{\delta}(s)$ by solving the minimization problem

$$
\min _{U \in H} J_{\alpha}(U), \quad J_{\alpha}(U)=\left\|(i \cdot)^{-\beta} U-F^{\delta}\right\|^{2}+\alpha\left\|\left(1+\cdot^{2}\right)^{\frac{p}{2}} U\right\|^{2}
$$


where again the dot is place-holder for the variable $s$. Hence, in the frequency domain the Tikhonov regularized solution $U_{\alpha}^{\delta}$ can be written in the form

$$
U_{\alpha}^{\delta}(s)=\frac{|s|^{\beta} e^{i \beta \operatorname{sgn}(s) \frac{x}{2}}}{1+\alpha\left(1+s^{2}\right)^{p}|s|^{2 \beta}} F^{\delta}(s) .
$$

The second summand in the denominator of (4.2) has a regularizing effect and damps high frequencies. The numerical computation of the regularized approximation $u_{\alpha}^{\delta}=$ $\mathcal{F}^{-1} U_{\alpha}^{\delta}$ can, for example, be done by discrete Fourier transforms.

By comparing (4.2) and (3.3) we see that $u_{\alpha}^{\delta}$ is the solution of the operator equation (1.1) with 'smoothed' data $f=f_{\alpha}^{\delta}$ where

$$
F_{\alpha}^{\delta}(s)=\frac{1}{1+\alpha\left(1+s^{2}\right)^{p}|s|^{2 \beta}} F^{\delta}(s),
$$

hence, a second way for the computation of $u_{\alpha}^{\delta}$ consists in executing the following steps:

(i) Given noisy data $f^{\delta}$, compute 'smoothed' data $f_{\alpha}^{\delta}=\mathcal{F}^{-1} F_{\alpha}^{\delta}$ by using discrete Fourier transforms where $F_{\alpha}^{\delta}$ is given by (4.3).

(ii) Given 'smoothed' data $f_{\alpha}^{\delta}$ from step (i), solve problem (1.1) with 'smoothed' data $f=f_{\alpha}^{\delta}$ to obtain $u_{\alpha}^{\delta}$.

In this way high frequency components in the data $f^{\delta}$ are filtered out properly. A similar regularization idea has bcen used in [8] where the data smoothing step (i) has been done by mollification techniques, and where order optimality has been achieved. In our following theorem we answer the question how to choose the regularization parameter $\alpha$ in (4.1) - (4.3), respectively, such that the Tikhonov regularized solution $u_{\alpha}^{\delta}=\mathcal{F}^{-1} U_{\alpha}^{\delta}$ is 'optimal' on the set $M_{p, E}$ given by (4.1).

Theorem 4.1. The Tikhonov regularized solution $u_{\alpha}^{\delta}=\mathcal{F}^{-1} U_{\alpha}^{\delta}$ with $U_{\alpha}^{\delta}$ given by (4.2) is 'optimal' on the set $M_{p, E}$ provided the regularization parameter $\alpha$ is chosen optimally by

$$
\alpha_{0}=\frac{\beta\left(1+r_{0}\right)}{p r_{0}}\left(\frac{\delta}{E}\right)^{2}
$$

where $r_{0}$ is the (unique) solution of equation (3.13). For $\delta \rightarrow 0$,

$$
\alpha_{0}=\frac{\beta}{p}\left(\frac{\delta}{E}\right)^{2}(1+o(1))
$$

holds. Furthermore, the optimal error estimate $\left\|u_{\alpha_{0}}^{\delta}-u\right\| \leq \omega(\delta)$ holds where $\omega(\delta)$ is given by (3.12).

Proof. From Theorem 2.3 it follows that the optimal regularization parameter $\alpha$ is given by (2.7) where $\varphi$ is given by (3.7). For functions $\varphi$ given in parameter representation $\lambda=\psi_{1}(r), \varphi=\psi_{2}(r)(0 \leq r<\infty)$ formula (2.7) attains the form

$$
\alpha=\frac{\dot{\psi}_{1}\left(r_{0}\right) \psi_{2}\left(r_{0}\right)}{\psi_{1}\left(r_{0}\right) \dot{\psi}_{2}\left(r_{0}\right)}\left(\frac{\delta}{E}\right)^{2}
$$

where $r_{0}$ is the (unique) solution of the equation $\psi_{1}(r) \psi_{2}(r)=\delta^{2} / E^{2}$. Using the special parameter representation (3.7) we obtain (4.4) and (3.13). The asymptotical expression for $\alpha_{0}$ follows directly from (4.4) and (3.13), and the optimal error estimate follows from Theorem 2.3 
In the second part of this section we consider the spectral method (2.9), apply it to problem (3.1) and show by applying Theorem 2.4 how to choose the regularization parameter such that this method is optimal on the set $M_{p, E}$.

For our fractional differentiation problem we obtain together with (3.3) and (3.4) that the spectral method (2.9) applied to problem (3.1) consists in the determination of a regularized approximation $U_{\alpha}^{\delta}$ according to

$$
U_{\alpha}^{\delta}(s)= \begin{cases}|s|^{\beta} e^{i \beta \operatorname{sgn}(s) \frac{\pi}{2}} F^{\delta}(s) & \text { for }|s|^{-2 \beta} \geq \alpha \\ \frac{1}{\alpha}|s|^{-\beta} e^{i \beta \operatorname{sgn}(s) \frac{\pi}{2}} F^{\delta}(s) & \text { for }|s|^{-2 \beta}<\alpha .\end{cases}
$$

The regularized solution (4.5) can also be rewritten in the form $U_{\alpha}^{\delta}=\hat{A}_{\beta}^{-1} F_{\alpha}^{\delta}$ with

$$
F_{\alpha}^{\delta}(s)= \begin{cases}F^{\delta}(s) & \text { for }|s|^{-2 \beta} \geq \alpha \\ \frac{1}{\alpha}|s|^{-2 \beta} F^{\delta}(s) & \text { for }|s|^{-2 \beta}<\alpha .\end{cases}
$$

Consequently, the numerical computation of $u_{\alpha}^{\delta}=\mathcal{F}^{-1} U_{\alpha}^{\delta}$ can also be carried out by the following two steps:

(i) Compute 'smoothed' data $f_{\alpha}^{\delta}=\mathcal{F}^{-1} F_{\alpha}^{\delta}$ by using discrete Fourier transforms.

(ii) Solve problem (1.1) with 'smoothed' data $f=f_{\alpha}^{\delta}$.

In our following theorem we answer the question how to choose the regularization parameter $\alpha$ in (4.5) or (4.6), respectively, such that the regularized solution $u_{\alpha}^{\delta}=\mathcal{F}^{-1} U_{\alpha}^{\delta}$ is 'optimal' on the set $M_{p, E}$ given by (4.1).

Theorem 4.2. The regularized solution $u_{\alpha}^{\delta}=\mathcal{F}^{-1} U_{\alpha}^{\delta}$ with $U_{\alpha}^{\delta}$ given by (4.5) is 'optimal' on the set $M_{p, E}$ provided the regularization parameter $\alpha$ is chosen optimally by

$$
\alpha_{0}=\frac{\beta\left(1+r_{0}\right)+p r_{0}}{p r_{0}^{\beta+1}}
$$

where $r_{0}$ is the (unique) solution of equation (3.13). For $\delta \rightarrow 0$,

$$
\alpha_{0}=\frac{\beta+p}{p}\left(\frac{\delta}{E}\right)^{\frac{2 \beta}{\beta+p}}(1+o(1))
$$

holds. Furthermore, the optimal error estimate $\left\|u_{\alpha_{0}}^{\delta}-u\right\| \leq \omega(\delta)$ holds with $\omega(\delta)$ given by (3.12).

Proof. From Theorem 2.4 it follows that the optimal regularization parameter $\dot{\alpha}$ is given by (2.10) with $\varphi$ from (3.7). For functions $\varphi$ given in parameter representation $\lambda=\psi_{1}(r), \varphi=\psi_{2}(r)\left(r \in \mathbb{R}^{+}\right)$formula (2.10) attains the form

$$
\alpha=\frac{\dot{\psi}_{1}\left(r_{0}\right) \psi_{2}\left(r_{0}\right)+\psi_{1}\left(r_{0}\right) \dot{\psi}_{2}\left(r_{0}\right)}{\dot{\psi}_{2}\left(r_{0}\right)}
$$

where $r_{0}$ is the (unique) solution of the equation $\psi_{1}(r) \psi_{2}(r)=\delta^{2} / E^{2}$. Using the special parameter representation (3.7) we obtain (4.7) and (3.13). Now the asymptotical expression for $\alpha_{0}$ follows from (4.7) together with (3.13), and the optimal error bound follows from Theorem 2.4 
In the third part of this section we consider the spectral method (2.11), apply it to problem (3.1) and show by applying Theorem 2.5 how to choose the regularization parameter such that this method is optimal on the set $M_{p, E}$.

For our fractional differentiation problem (1.1) we obtain together with (3.3) and (3.4) that the spectral method (2.11) consists in the determination of a regularized approximation $U_{a}^{\delta}$ according to

$$
U_{\alpha}^{\delta}(s)= \begin{cases}|s|^{\beta} e^{i \beta \operatorname{sgn}(s) \frac{\pi}{2}} F^{\delta}(s) & \text { for }|s|^{-2 \beta} \geq \alpha \\ \frac{1}{\sqrt{\alpha}} e^{i \beta \operatorname{sgn}(s) \frac{\pi}{2}} F^{\delta}(s) & \text { for }|s|^{-2 \beta}<\alpha .\end{cases}
$$

For this method we obtain the following optimality results whose proof follows from Theorem 2.5 and can be carried out according to that of Theorem 4.2.

Theorem 4.3. The regularized solution $u_{\alpha}^{\delta}=\mathcal{F}^{-1} U_{\alpha}^{\delta}$ with $U_{\alpha}^{\delta}$ given by (4.8) is 'optimal' on the set $M_{p, E}$ provided the regularization parameter $\alpha$ is chosen optimally by

$$
\alpha_{0}=r_{0}^{-\beta}\left[\frac{\beta\left(1+r_{0}\right)+p r_{0}}{p r_{0}}\right]^{2}
$$

where $r_{0}$ is the (unique) solution of equation (3.13). For $\delta \rightarrow 0$,

$$
\alpha_{0}=\left(\frac{\beta+p}{p}\right)^{2}\left(\frac{\delta}{E}\right)^{\frac{2 p}{\beta+p}}(1+o(1))
$$

holds. Furthermore, the optimal error estimate $\left\|u_{\alpha_{0}}^{\delta}-u\right\| \leq \omega(\delta)$ holds with $\omega(\delta)$ given by (3.12).

\section{Optimal approximations in Sobolev scales}

In this section we consider the fractional differentiation problem (1.1) of Section 1 and suppose as in the previous sections that the data and the (unknown) solution satisfy the two assumptions

(A1) $\left\|f-f^{\delta}\right\| \leq \delta$

(A2) $\|u\|_{p} \leq E$

where $\|\cdot\|_{p}$ is defined according to (1.2). In contrast to Sections 3 and 4 we are here interested in best possible error bounds not with respect to the $H$-norm, but with respect to the stronger $H^{q}$-norm $(0 \leq q \leq p)$. In addition, we are interested in the construction of special regularization methods that guarantee these best possible error bounds with respect to the $H^{q}$-norm. Hence, we will answer the following two questions:

(Q1) Which best possible accuracy can be obtained (with respect to the $H^{q}$-norm, $0 \leq q<p$ ) for identifying $u$ from $f^{\delta}$ under assumptions (A1) and (A2)?

(Q2) In which way do we have to construct special regularization methods $R_{\alpha}$ : $H \rightarrow H^{q}$ such that the corresponding regularized approximations $u_{\alpha}^{\delta}=R_{\alpha} f^{\delta}$ guarantee this best possible accuracy with respect to the $H^{q}$-norm? 
Let us start with the discussion of question (Q1). We formulate our fractional differentiation problem (1.1) as an operator equation

$$
A_{\beta, q} u=f, \quad A_{\beta, q}: H^{q} \rightarrow H
$$

where $A_{\beta, q}$ denotes the restriction of the operator $A_{\beta}$ to $H^{q}$. We introduce a Hilbert scale $\left(\hat{H}^{r}\right)_{r \in \mathbb{R}+}$ in the frequency domain according to

$$
\widehat{H}^{0}=\widehat{H}=L^{2}(\mathbb{R}), \quad \widehat{H}^{r}=\left\{V \in \widehat{H} \mid\|V\|_{r}<\infty\right\}
$$

with

$$
\|V\|_{r}=\left\|\left(1+s^{2}\right)^{\frac{r}{2}} V(s)\right\|_{L^{2}(\mathbb{R})} \quad\left(r \in \mathbb{R}^{+}\right)
$$

as norm in $\hat{H}^{r}$. Furthermore, let us transform equation (5.1) into an equivalent operator equation

$$
\widehat{A}_{\beta, q} U=F, \quad \widehat{A}_{\beta, q}: \widehat{H}^{q} \rightarrow \widehat{H}
$$

in the frequency domain. Then it is obvious that $\hat{A}_{\beta, q}=|s|^{-\beta} e^{-i \beta \operatorname{sgn}(s) \frac{x}{2}}$ (cf. (3.3)). Due to the relations

$$
\begin{aligned}
\left(\widehat{A}_{\beta, q} V_{1}, V_{2}\right)_{0} & =\left(V_{1},|s|^{-\beta} e^{i \beta \operatorname{sgn}(s) \frac{\pi}{2}} V_{2}\right)_{0} \\
& =\left(V_{1},\left(1+s^{2}\right)^{-q}|s|^{-\beta} e^{i \beta \operatorname{sgn}(s) \frac{\pi}{2}} V_{2}\right)_{q}
\end{aligned}
$$

we obtain $\widehat{A}_{\beta, q}^{*}=\left(1+s^{2}\right)^{-q}|s|^{-\beta} e^{i \beta \text { sgn(s) } \frac{\pi}{2}}$. which shows us that $\widehat{A}_{\beta, q}^{*} \widehat{A}_{\beta, q}: \widehat{H}^{q} \rightarrow \widehat{H}^{q}$ is given by

$$
\widehat{A}_{\beta, q}^{*} \widehat{A}_{\beta, q}=\left(1+s^{2}\right)^{-q}|s|^{-2 \beta} \text {. }
$$

This representation enables us to reformulate our quantitative a priori information (A2) into an equivalent condition in the frequency domain. We use (1.2) and (5.2), and obtain that (A2) is equivalent to the condition

$$
\left\|\left(1+s^{2}\right)^{\frac{p-q}{2}} U(s)\right\|_{q} \leq E .
$$

Consequently, using (5.3) and (5.4) we obtain that our quantitative a priori information (A2) can be rewritten in the equivalent form

$$
U \in \widehat{M}_{\varphi, E}=\left\{U \in \widehat{H}^{q} \mid\left\|\left[\varphi\left(\widehat{A}_{\beta, q}^{*} \widehat{A}_{\beta, q}\right)\right]^{-\frac{1}{2}} U\right\|_{q} \leq E\right\}
$$

where $\varphi$ is given (in parameter representation) by

$$
\left.\begin{array}{l}
\lambda=(1+r)^{-q} r^{-\beta} \\
\varphi=(1+r)^{q-p}
\end{array}\right\} \quad\left(r \in \mathbb{R}^{+}\right) .
$$

The function $\varphi$ given (in parameter representation) by (5.6) possesses the following properties which can be shown along the lines of the proof of Proposition 3.2. 
Proposition 5.1. If $0 \leq q<p$, then the function $\varphi$ defined by (5.6) is continuous and satisfies the following properties:

(i) $\lim _{\lambda \rightarrow 0} \varphi(\lambda)=0$.

(ii) $\varphi$ is strictly monotonically increasing.

(iii) $\rho(\lambda)=\lambda \varphi^{-1}(\lambda)$ is strictly monotonically increasing as well as strictly convex and possesses the parameter representation

$$
\left.\begin{array}{l}
\lambda=(1+r)^{q-p} \\
\rho=r^{-\beta}(1+r)^{-p}
\end{array}\right\} \quad(0 \leq r<\infty) .
$$

(iv) $\rho^{-1}$ is strictly monotonically increasing and possesses the parameter representation

$$
\left.\begin{array}{rl}
\lambda & =r^{-\beta}(1+r)^{-p} \\
\rho^{-1} & =(1+r)^{q-p}
\end{array}\right\} \quad(0 \leq r<\infty) .
$$

(v) For the inverse function $\rho^{-1}$ of $\rho$,

$$
\rho^{-1}(\lambda)=\lambda^{\frac{p-q}{p+\beta}}(1+o(1)) \quad \text { for } \lambda \rightarrow 0
$$

holds.

Now we are ready to discuss question (Q1) concerning the magnitude of the best possible worst case error

$$
\omega_{q}(\delta)=\inf _{R: H \rightarrow H_{q}} \sup \left\{\left\|R f^{\delta}-u\right\|_{q} \mid f^{\delta} \in H, u \in M_{p, E},\left\|f-f^{\delta}\right\| \leq \delta\right\}
$$

with $M_{p, E}$ given by (1.4) that shows us which best possible accuracy can be obtained (with respect to the $H^{q}$-norm) for identifying $u$ from $f^{\delta}$ under assumptions (A1) and (A2). We use the fact that $\omega_{q}(\delta)=\widehat{\omega}_{q}(\delta)$ where

$$
\widehat{\omega}_{q}(\delta)=\inf _{\widehat{R}: \widehat{H} \rightarrow \widehat{H}^{\natural}} \sup \left\{\left\|\widehat{R} F^{\delta}-U\right\|_{q} \mid F^{\delta} \in \widehat{H}, U \in \widehat{M}_{\varphi, E},\left\|F-F^{\delta}\right\| \leq \delta\right\}
$$

with $\widehat{M}_{\varphi, E}$ given by (5.5) and $\varphi$ given by (5.6), apply Theorem 2.2 with $A: H_{1} \rightarrow H_{2}$ replaced by $\widehat{A}_{\beta, q}: \widehat{H}^{q} \rightarrow \widehat{H}$ and Proposition 5.1 , proceed according to the proof of Theorem 3.3 and obtain

Theorem 5.2. Let $\delta^{2} / E^{2}<\infty, 0 \leq q<p$ and $\beta>0$. Then the stability result

$$
\begin{aligned}
\omega_{q}(\delta) & =E\left(1+r_{0}\right)^{\frac{q-p}{2}} \\
& =E^{\frac{q+\beta}{p+\beta}} \delta^{\frac{p-q}{p+\beta}}(1+o(1)) \quad \text { for } \delta \rightarrow 0
\end{aligned}
$$

holds (Hölder stability) where $r_{0}$ is the (unique) solution of equation (3.13).

In the second part of this section we construct special regularization methods $R_{\alpha}$ : $H \rightarrow H^{q}$ that are 'optimal' in the sense that the corresponding regularized solutions $u_{\alpha}^{\delta}=R_{\alpha} f^{\delta}$ guarantee 'optimal' error bounds $\left\|u_{\alpha}^{\delta}-u\right\|_{q} \leq \omega_{q}(\delta)$ where $\omega_{q}(\delta)$ is given in Theorem 5.2. We apply Theorems $2.3 \cdot 2.5$, proceed along the lines of Section 4 and obtain the following results. 
Theorem 5.3. Let $\delta^{2} / E^{2}<\infty, 0 \leq q<p$ and $\beta>0$. Then the Tikhonov regularized solution $u_{\alpha}^{\delta}=\mathcal{F}^{-1} U_{\alpha}^{\delta}$ with $U_{\alpha}^{\delta}$ given by (4.2) satisfies the optimal error estimate $\left\|u_{\alpha}^{\delta}-u\right\|_{q} \leq \omega_{q}(\delta)$ with $\omega_{q}(\delta)$ given by $(5.8)$ provided the regularization parameter $\alpha=\alpha_{0}$ is chosen optimally by

$$
\alpha_{0}=\frac{\beta\left(1+r_{0}\right)+q r_{0}}{(p-q) r_{0}}\left(\frac{\delta}{E}\right)^{2}
$$

where $r_{0}$ is the (unique) solution of equation (3.13). For $\delta \rightarrow 0$ the asymptotical representation

$$
\alpha_{0}=\frac{\beta+q}{p-q}\left(\frac{\delta}{E}\right)^{2}(1+o(1))
$$

holds.

Theorem 5.4. Let $\delta^{2} / E^{2}<\infty, 0 \leq q<p$ and $\beta>0$. Then the regularized solution $u_{\alpha}^{\delta}=\mathcal{F}^{-1} U_{\alpha}^{\delta}$ with $U_{\alpha}^{\delta}$ given by

$$
U_{\alpha}^{\delta}(s)= \begin{cases}|s|^{\beta} e^{i \beta \operatorname{sgn}(s) \frac{\pi}{2}} F^{\delta}(s) & \text { for }\left(1+s^{2}\right)^{-q}|s|^{-2 \beta} \geq \alpha \\ \frac{1}{\alpha}\left(1+s^{2}\right)^{-q}|s|^{-\beta} e^{i \beta \operatorname{sgn}(s) \frac{\pi}{2} F^{\delta}(s)} & \text { for }\left(1+s^{2}\right)^{-q}|s|^{-2 \beta}<\alpha\end{cases}
$$

satisfies the optimal error estimate $\left\|u_{\alpha}^{\delta}-u\right\|_{q} \leq \omega_{q}(\delta)$ with $\omega_{q}(\delta)$ given by (5.8) provided the regularization parameter $\alpha=\alpha_{0}$ is chosen optimally by

$$
\alpha_{0}=\frac{\beta\left(1+r_{0}\right)+p r_{0}}{(p-q)\left(1+r_{0}\right)^{q} r_{0}^{\beta+1}}
$$

where $r_{0}$ is the (unique) solution of equation (3.13). For $\delta \rightarrow 0$ the asymptotical representation

$$
\alpha_{0}=\frac{\beta+p}{p-q}\left(\frac{\delta}{E}\right)^{\frac{2(q+\beta)}{p+\beta}}(1+o(1))
$$

holds.

Theorem 5.5. Let $\delta^{2} / E^{2}<\infty, 0 \leq q<p$ and $\beta>0$. Then the regularized solution $u_{\alpha}^{\delta}=\mathcal{F}^{-1} U_{\alpha}^{\delta}$ with $U_{\alpha}^{\delta}$ given by

$$
U_{\alpha}^{\delta}(s)= \begin{cases}|s|^{\beta} e^{i \beta \operatorname{sgn}(s) \frac{\pi}{2}} F^{\delta}(s) & \text { for }\left(1+s^{2}\right)^{-q}|s|^{-2 \beta} \geq \alpha \\ \frac{1}{\sqrt{\alpha}}\left(1+s^{2}\right)^{-q / 2} e^{i \beta \operatorname{sgn}(s) \frac{\pi}{2}} F^{\delta}(s) & \text { for }\left(1+s^{2}\right)^{-q}|s|^{-2 \beta}<\alpha\end{cases}
$$

satisfies the optimal error estimate $\left\|u_{\alpha}^{\delta}-u\right\|_{q} \leq \omega_{q}(\delta)$ with $\omega_{q}(\delta)$ given by $(5.8)$ provided the regularization parameter $\alpha=\alpha_{0}$ is chosen optimally by

$$
\alpha_{0}=\left(1+r_{0}\right)^{-q} r_{0}^{-\beta}\left[\frac{\beta\left(1+r_{0}\right)+p r_{0}}{(p-q) r_{0}}\right]^{2}
$$

where $r_{0}$ is the (unique) solution of equation (3.13). For $\delta \rightarrow 0$ the asymptotical representation

$$
\alpha_{0}=\left(\frac{\beta+p}{p-q}\right)^{2}\left(\frac{\delta}{E}\right)^{\frac{2(q+\beta)}{p+\beta}}(1+o(1))
$$

holds. 


\section{References}

[1] Baumeister, J.: Stable Solution of Inverse Problems. Braunschweig: Vieweg 1987.

[2] Engl, H. W., Hanke, M. and A. Neubauer: Regularization of Inverse Problems. Dordrecht: Kluwer 1996.

[3] Gerlach, W. and L. v. Wolfersdorf: On approximate computation of the values of the normal derivative of solutions to linear partial differential equations of second order with application to Abel's integral equation. Z. Ang. Math. Mech. (ZAMM) 66 (1986), 31 36.

[4] Gorenflo, R., Medvedeva, T. and B. Rubin: Locally controllable regularization of Abel integral equations via operator complementation. J. Inv. Ill-Posed Probl. 5 (1997), 427 436.

[5] Gorenflo, R. and B.'Rubin: Locally controllable regularization of fractional derivatives. Inverse Problems 10 (1994), 881 - 893.

[6] Gorenflo, R. and S. Vessella: Abel Integral Equations: Analysis and Applications. Berlin: Springer 1991.

[7] Groetsch, C. W.: The Theory of Tikhonov Regularization for Fredholm Equations of the First Kind. Boston: Pitman 1984.

[8] Haó, D. N., Reinhardt, H.-J. and F. Seiffarth: Stable numerical fractional differentiation by mollification. Numer. Funct. Anal, and Opt. 15 (1994), 635 - 659.

[9] Haó, D. N., Reinhardt, H.-J. and A. Schneider: Stable approximation of fractional derivatives of rough functions. BIT 35 (1995), $488-503$.

[10] Hofmann, B.: Regularization for Applied Inverse and Jll-Posed Problems (Teubner-Texte zur Mathematik: Vol 85). Leipzig: B. G. Teubner 1986.

[11] Krein, S. and Y. I. Petunin: Scales of Banach spaces. Russian Math. Surveys 21 (1966), $85-159$.

[12] Louis, A.: Inverse und schlecht gestellte Probleme. Stuttgart: B. G. Teubner 1989.

[13] Mair, B. A.: Tikhonov regularization for finitely and infinitely smoothing operators. SIAM J. Math. Anal. 25 (1994), 135 - 147.

[14] Plato, R.: The Galerkin scheme for Lavrentiev's $m$-times iterated method to solve linear accretive Volterra integral equations of the first kind. BIT 37 (1997), $404-423$.

[15] Plato, R.: Resolvent estimates for Abel integral operators and the regularization of associated first kind integral equations. J. Int. Equ. Appl. 9 (1997), $253-278$.

[16] Samko, S. G., Kilbas, A. A. and O. I. Marichev: Fractional Integrals and Derivatives: Theory and Applications. Switzerland: Gordon and Breach Sci. Publ. 1993.

[17] Schröter, T. and U. Tautenhahn: On the optimality of regularization methods for solving linear ill-posed problems. Z. Anal. Anw. 13 (1994), 697 - 710.

[18] Seidman, T. I.: 'Optimal Filtering' for some ill-posed problems. In: Wave Propagation and Inversion (eds.: W. Fitzgibbon and M. Wheeler). Philadelphia: SIAM 1992, pp. 108 $-123$.

[19] Tautenhahn, U.: Optimality for linear ill-posed problems under general source conditions. Numer. Funct. Anal. and Optimiz. 19 (1998), 377 - 398.

[20] 'Tautenhahn, U.: Optimal stable solution of Cauchy problems for elliptic equations. Z. Anal. Anw. 15 (1996), 961 - 984.

[21] Tautenhahn, U.: Optimal stable approximations for the sideways heat equation. J. Inv. Ill-Posed Probl. 5 (1997), 287 - 307. 
[22] Tikhonov, A. N. and V. Y. Arsenin: Solution of Ill-Posed Problems. New York: Wiley 1977.

(23) Tuan, V. K. and R. Gorenflo: On the regularization of fractional differentiation of arbitrary positive order. Numer. Funct. Anal. and Opt. 15 (1994), $695-711$.

[24] Vainikko, G. M.: On the optimality of methods for ill-posed problems. Z. Anal. Anw. 6 (1987), 351 - 362.

[25] Vainikko, G. M. and A. Y. Veretennikov: Iteration Procedures in Ill-Posed Problems (in Russian). Moscow: Nauka 1986.

Received 30.06.1998 